University of Nebraska - Lincoln

DigitalCommons@University of Nebraska - Lincoln

Timothy J. Gay Publications

Research Papers in Physics and Astronomy

2014

Chirally Sensitive Electron-Induced Molecular Breakup and the Vester-Ulbricht Hypothesis

J. M. Dreiling

Timothy J. Gay

Follow this and additional works at: https://digitalcommons.unl.edu/physicsgay

Part of the Atomic, Molecular and Optical Physics Commons, and the Other Physics Commons

This Article is brought to you for free and open access by the Research Papers in Physics and Astronomy at DigitalCommons@University of Nebraska - Lincoln. It has been accepted for inclusion in Timothy J. Gay Publications by an authorized administrator of DigitalCommons@University of Nebraska - Lincoln. 


\title{
Chirally Sensitive Electron-Induced Molecular Breakup and the Vester-Ulbricht Hypothesis
}

\author{
J. M. Dreiling* and T. J. Gay \\ Jorgensen Hall, University of Nebraska, Lincoln, Nebraska 68588-0299, USA
}

(Received 6 July 2014; published 12 September 2014)

\begin{abstract}
We have studied dissociative electron attachment in sub-eV collisions between longitudinally polarized electrons and chiral bromocamphor molecules. For a given target enantiomer, the dissociative $\mathrm{Br}$ anion production depends on the helicity of the incident electrons, with an asymmetry that depends on the electron energy and is of order $3 \times 10^{-4}$. The existence of chiral sensitivity in a well-defined molecular breakup reaction demonstrates the viability of the Vester-Ulbrict hypothesis, namely, that the longitudinal polarization of cosmic beta radiation was responsible for the origins of biological homochirality.
\end{abstract}

DOI: 10.1103/PhysRevLett.113.118103

PACS numbers: $34.80 . \mathrm{Ht}, 34.80 . \mathrm{Nz}, 82.30 . \mathrm{Lp}, 87.23 . \mathrm{Kg}$

All molecular forms of life possess a chiral asymmetry, with amino acids and sugars found, respectively, in $L$ - and $D$-enantiomers only [1]. The primordial origin of this enantiomeric excess is unknown. One possible explanation is given by the Vester-Ulbricht hypothesis, which suggests that left-handed electrons present in beta radiation, produced by parity-violating weak decays, interacted with biological precursor molecules, preferentially destroying one of their two enantiomers $[2,3]$. Vester and Ulbricht's ansatz suggested that the circularly polarized bremsstrahlung produced by chiral (longitudinally spin-polarized) beta radiation would preferentially photolyze chiral molecules of one handedness [3]. A second possibility is that the electrons themselves, in direct interaction with the chiral molecules, might preferentially destroy one enantiomer.

Experiments searching for chirally sensitive chemical breakup reactions initiated by beta radiation or its equivalent - beams of longitudinally polarized electrons produced in the laboratory-have been plagued by negative results and/or irreproducibility [4-8]. However, several experiments have appeared to show chiral sensitivity. Some chiral amino acids are preferentially racemized by radiation from ${ }^{14} \mathrm{C}$ and ${ }^{60} \mathrm{Co}[9,10]$. Chirality-dependent decarboxylation in ${ }^{14} \mathrm{C}$-seeded leucine has been observed [11]. Unfortunately, the mechanism for these effects and, specifically, whether they are induced by the beta rays themselves or subsequent polarized bremsstrahlung, is unclear. In a recent experiment using electrons photoemitted by $\mathrm{x}$ rays incident on a magnetized iron substrate, large chirally sensitive chemical changes were reported for $(R, S)$-2-butanol deposited on the iron's surface [12]. A specific reaction channel for the electron-molecule interaction was not experimentally identified. The interested reader is referred to several comprehensive, critical reviews of the literature in this area $[5,6,13]$.

Here, we report the observation of a chirally sensitive molecular breakup process in the gas phase. This allows us to unambiguously identify the chirality dependence of the reaction, without the influence of condensed matter effects that can complicate the interpretation of experimental results. With an unoriented gas-phase target and cylindrically symmetric detection of reaction products, the entrance and exit reaction channels can be identified, and any asymmetries that are observed must result from the inherent chirality of the target molecules. The use of longitudinally polarized free electrons mimics an experiment with beta rays, but with controllable energy and without the complicating possibility of photolysis due to bremsstrahlung. We searched for these effects in dissociative electron attachment [14] (DEA) in 3-bromocamphor:

$$
\mathrm{e}^{-}+\mathrm{C}_{10} \mathrm{H}_{15} \mathrm{BrO} \rightarrow \mathrm{C}_{10} \mathrm{H}_{15} \mathrm{O}^{\bullet}+\mathrm{Br}^{-} \text {. }
$$

DEA is a good candidate for such a search because it occurs through a resonant channel in which a molecular anion is temporarily produced. The formation of a temporary negative ionic state allows the incident electron and the molecular target to "sample each others chirality" during the compound state's resonant lifetime, enhancing any possible chiral sensitivity of the breakup reaction [15]. Moreover, bromocamphor has been measured to have a very large DEA cross section at near-zero incident electron energy [16]; low incident electron velocities should enhance any "chirality sampling" effects. While cosmic beta rays have high $(\sim \mathrm{keV})$ initial energies, they must ultimately slow to low energy before stopping. Coulombic interactions associated with stopping power would not generally depolarize these electrons completely. The essential role played by low-energy achiral electrons in biologically important bond-breaking processes has been demonstrated in the experiments of Sanche and others [17-20].

Our experimental apparatus has four main components: an active-feedback optical system, a spin-polarized electron source, a target chamber, and an optical electron polarimeter. The spin-polarized electron source, shown in Fig. 1, is based on photoemission from negative-electron-affinity 


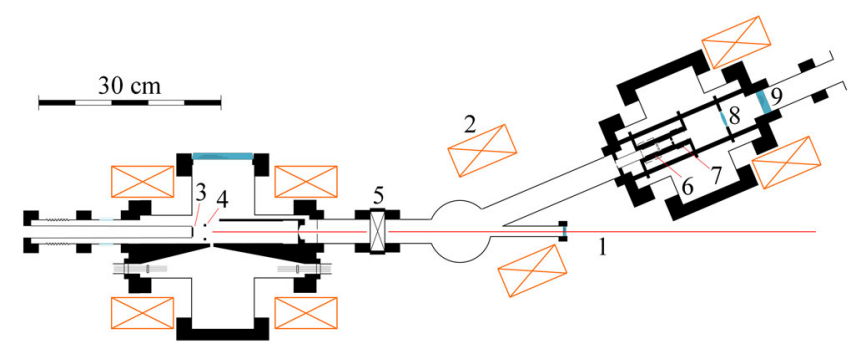

FIG. 1 (color online). Polarized electron source and target chamber. (1) Laser beam from active-feedback system for the GaAs source, (2) solenoidal guiding magnets, (3) GaAs photocathode, (4) NEA activation cesiators, (5) gate valve, (6) chiral target cell (see also Fig. 2), (7) optical polarimeter target cell, (8) fluorescence collection lens, (9) window for the optical polarimeter. The bend in the apparatus eliminates the line of sight between the target cell and the GaAs crystal to minimize chemical contamination of the NEA photocathode.

(NEA) GaAs [21]; the electron's longitudinal polarization is determined by the helicity of incident laser light. The laser's circular polarization is reversed by the optical system, which also allows for the reduction of instrumental asymmetries via active feedback similar to that discussed in Fabrikant et al. [22]. Following extraction from the source, the electron beam is magnetically guided around a bend and into the target (Fig. 1). The target chamber is arranged to allow electron polarization measurement with an optical polarimeter [23]; our electron polarization is typically $\sim 30 \%$.

During a chiral asymmetry measurement, molecules of a given handedness are admitted to the target cell (TC) (Figs. 1 and 2). An anion current is measured on the isolated inner target cell wall, and the unscattered electron beam current is detected on the Faraday cup (FC) following the target cell. Scattered electrons are largely prevented from reaching the walls of the target and are instead guided out of the cell by a $\sim 15 \mathrm{mT}$ longitudinal magnetic field. Electron-spin-dependent asymmetries are determined by measuring these currents using a lock-in amplifier referenced to the frequency of electron spin reversal.

To test the accuracy of our methods, we first did experiments investigating the spin-polarized electron transmission through a chirally pure bromocamphor vapor by measuring currents on the Faraday cup. This experiment had previously been done by the group of Kessler [24,25]. Our results, though more sparsely distributed in energy, are in good qualitative agreement with theirs and demonstrated that our apparatus had the sensitivity required to measure chirally dependent electron asymmetries less than $10^{-4}$.

We confirmed our apparatus's ability to detect a DEA signal on the target cell walls at a near-zero incident electron energy by investigating molecules with DEA cross sections that peak near $0 \mathrm{eV}: \mathrm{CHCl}_{3}$ and $\mathrm{C}_{6} \mathrm{H}_{4} \mathrm{Cl}_{2}$. Our measurements were in qualitative agreement with those of Aflatooni and Burrow [16,26], both in terms of the total
DEA cross sections and the electron energies corresponding to the peaks of the DEA cross sections. Thus, we are confident that we are detecting a negative ion current formed in the DEA process. There exists the possibility of scattered electrons contributing to the signal; this is discussed in more detail below.

To take DEA asymmetry data, chirally pure bromocamphor was admitted to the target cell until the incident electron beam of $\sim 0 \mathrm{eV}$ energy was attenuated by about $50 \%$. This corresponded to a bromocamphor pressure of 0.5-1.0 mTorr as measured by a capacitance manometer. Multiple scattering has a negligible effect on our measured asymmetries because it does not significantly alter the electron beam polarization. The electron helicity was reversed at a frequency of $\sim 210 \mathrm{~Hz}$, and the DEA current asymmetry associated with the helicity reversal, $a=(I \uparrow-I \downarrow) /(I \uparrow+I \downarrow)$, was monitored for $\sim 3$ minutes. Here, $I \uparrow(I \downarrow)$ is the current measured on the target cell wall for spin-forward (spin-backward) electrons. Thus, $a_{L}\left(a_{R}\right)$ is the electron-helicity-dependent component of the DEA signal for the $L$ - and $R$-enantiomers of bromocamphor, respectively. The chirality of the gas was then switched and data collected again. A final asymmetry value $A$ was calculated using

$$
A=a_{L}-a_{R}=\left[\frac{I \uparrow-I \downarrow}{I \uparrow+I \downarrow}\right]_{L}-\left[\frac{I \uparrow-I \downarrow}{I \uparrow+I \downarrow}\right]_{R} .
$$

This formulation is used to compensate for persistent instrumental asymmetries that are common between the measurements for both enantiomers. At each energy, $A$ was measured $\sim 10$ times and an average was found after applying Chauvenet's criteria [27] to the data.

Figure 3 shows the DEA signal and its asymmetry for a range of electron energies near $0 \mathrm{eV}$. The energy scale is linear in the retarding voltage placed on the target cell, with $0 \mathrm{eV}$ corresponding to the peak in the derivative of the current transmitted to the Faraday cup with no target gas. Thus, "negative" energies correspond to the high energy tail of the incident electron beam, which had a nominally Gaussian profile and a FWHM energy width of $\sim 0.6 \mathrm{eV}$. In the energy range considered, the average incident electron kinetic energy in the target varies monotonically but nonlinearly with the abscissa value of retarding potential. At the most positive values, the beam's FWHM is the full $0.6 \mathrm{eV}$; at increasingly negative potentials, its energy spread in the target region approaches zero, as does its average kinetic energy.

The nonzero values of $A$ we measure indicate an electron-helicity-dependent breakup of chiral molecules. As such, they connect the universal chiral asymmetry of the weak force, which produces helical beta radiation, to a handedness-specific molecular breakup process, demonstrating the viability of the Vester-Ulbricht hypothesis. 


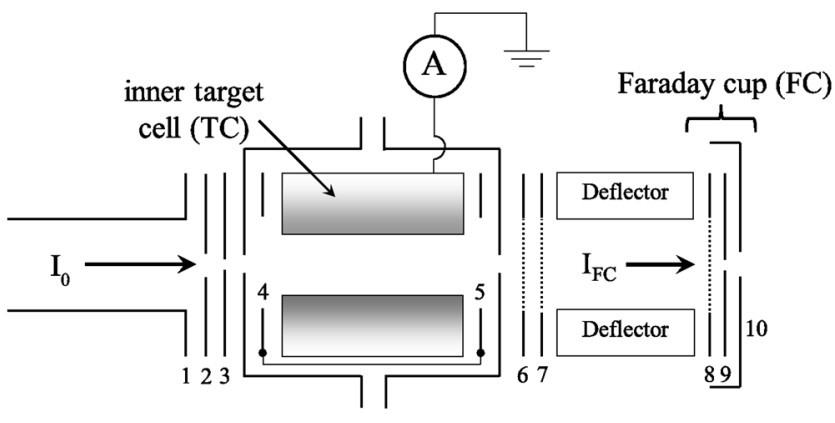

FIG. 2. Detail of the target vapor cell, showing the incident $\left(I_{0}\right)$ and transmitted $\left(I_{\mathrm{FC}}\right)$ electron beams, the target cell structure, lens elements 1-10, and the Faraday cup assembly used to measure the transmitted beam.

As a systematic check, the data were taken using two different settings of the quarter-wave plate that polarizes the laser light. This flips the sign of the electron polarization for a given optical configuration. We also made an asymmetry measurement using two samples of racemic bromocamphor. Data were taken exactly as before, with $a$ recorded for each of the samples, and $A$ calculated by finding the difference. (In this situation, the subscripts are arbitrary.) This measurement yields asymmetries consistent with zero.

In a total ion collection apparatus such as ours (Fig. 2), it is not possible to unambiguously identify the negative current measured on the target cell walls as being due solely to DEA product anions, because of contributions from
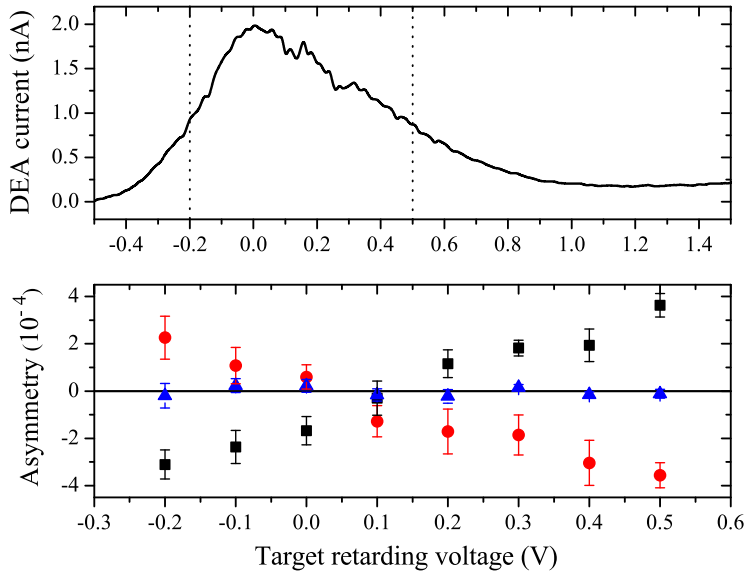

FIG. 3 (color online). Top: DEA current, with the range of energies where asymmetry data were taken indicated by the dotted vertical lines. Bottom: The asymmetry in DEA current as a function of target cell retarding voltage. Squares and circles represent opposite settings of the quarter-wave plate that circularly polarizes the laser light, which should give asymmetry measurements of opposite signs. A positive sign for the data represented as squares corresponds to a positive sign for the data of Nolting et al. [25] with (-) bromocamphor. The triangles indicate data taken with racemic bromocamphor. Uncertainties are determined by taking the standard deviation of the mean of the individual asymmetry measurements. electrons scattered, e.g., from beam apertures. This problem is exacerbated as the beam energy approaches zero. The longitudinal magnetic field should, in principle, eliminate this problem, but in reality it only serves to reduce it to a manageable level. To determine the extent of such scattered-electron contributions to the signal current, we performed a systematic study. This was done by comparing signals for both bromocamphor and nitrogen targets. While bromocamphor has a substantial DEA cross section in the energy range investigated, nitrogen does not. Therefore, the detected current with nitrogen provides a measure of the scattered-electron collection efficiency of the inner target cell.

We denote the current transmitted to the Faraday cup as $I_{\mathrm{FC}}$, while $I_{\mathrm{TC}}$ is the current detected on the inner target cell wall, and $I_{0}$ represents the electron current incident upon the target gas. Because the beam-defining aperture (element 3 in Fig. 2) is upstream of the target cell, it is reasonable to take $I_{0}$ to be the current detected by the Faraday cup with no target gas present: $I_{0} \equiv I_{\mathrm{FC}}(P=0)$.

In order to measure a true DEA signal, we should not detect a significant proportion of scattered electrons on the inner target cell (TC in Fig. 2). The ratio of the current detected on the inner target cell wall divided by the attenuated current, $I_{\mathrm{TC}} /\left(I_{0}-I_{\mathrm{FC}}\right)$, is plotted verses attenuation at $0 \mathrm{eV}$ in Fig. 4. As can be seen, a significant proportion of the attenuated current is detected for bromocamphor but not for nitrogen, suggesting that $I_{\mathrm{TC}}$ is predominantly due to the anions produced by DEA.

We assume that all attenuated current is due either to scattered electrons or DEA anions. The fraction of the attenuated current that is detected on the inner target cell wall is related to the proportion of electrons scattered out of the Faraday cup, $P_{\mathrm{s}}$, and of anions from DEA, $P_{\mathrm{DEA}}$, by the equation

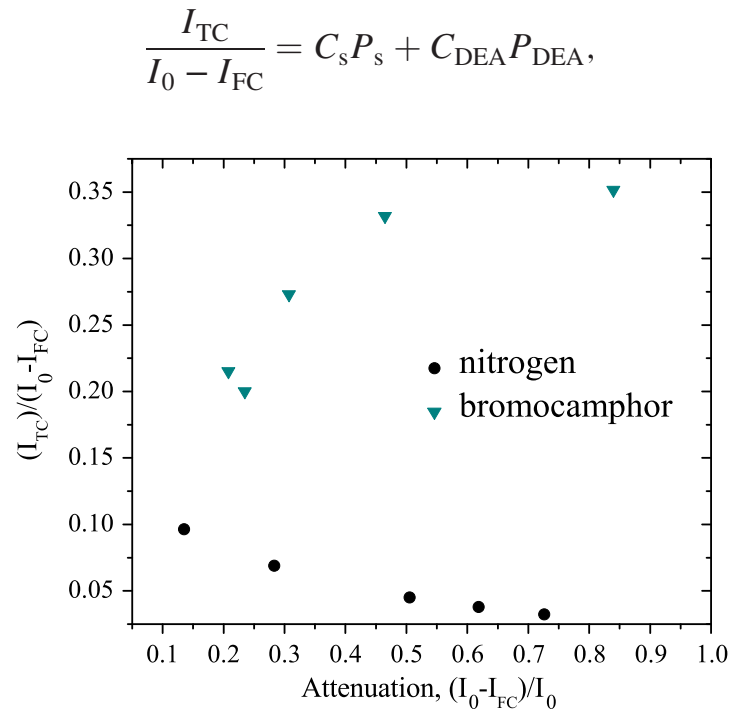

FIG. 4 (color online). The fraction of the attenuated current that is detected on the inner target cell walls. 
where $C_{\mathrm{s}}$ and $C_{\mathrm{DEA}}$ are the efficiencies for detecting scattered electrons and DEA anions, respectively, on the inner target cell. Because of the assumption that all of the current attenuated by bromocamphor is either scattered electrons or produces DEA, we have

$$
P_{\mathrm{DEA}}+P_{\mathrm{s}}=1 \text {. }
$$

The DEA cross section for nitrogen is essentially zero, meaning that $P_{\mathrm{s}}=1$ and

$$
C_{\mathrm{s}} \approx\left[\frac{I_{\mathrm{TC}}}{I_{0}-I_{\mathrm{FC}}}\right]_{\mathrm{N}_{2}},
$$

which gives

$$
\left[\frac{I_{\mathrm{TC}}}{I_{0}-I_{\mathrm{FC}}}\right]_{\mathrm{Br}}=\left[\frac{I_{\mathrm{TC}}}{I_{0}-I_{\mathrm{FC}}}\right]_{\mathrm{N}_{2}} \times P_{\mathrm{s}}+C_{\mathrm{DEA}}\left(1-P_{\mathrm{s}}\right) .
$$

Thus,

$$
P_{\mathrm{S}}=\frac{\left[\frac{I_{\mathrm{TC}}}{I_{0}-I_{\mathrm{FC}}}\right]_{\mathrm{Br}}-C_{\mathrm{DEA}}}{\left[\frac{I_{\mathrm{TC}}}{I_{0}-I_{\mathrm{FC}}}\right]_{\mathrm{N}_{2}}-C_{\mathrm{DEA}}},
$$

and the component of the DEA signal due to scattered electrons measured on the inner target cell wall is given by

$$
\frac{C_{\mathrm{S}} P_{\mathrm{s}}}{C_{\mathrm{DEA}} P_{\mathrm{DEA}}} \approx \frac{\left[\frac{I_{\mathrm{TC}}}{I_{0}-I_{\mathrm{FC}}}\right]_{\mathrm{N}_{2}} P_{\mathrm{s}}}{C_{\mathrm{DEA}}\left(1-P_{\mathrm{s}}\right)} \equiv \alpha .
$$

Data for the asymmetry measurements were mostly taken with $50 \%$ attenuation, so we will consider that case. Using Eq. (7) and our results for $I_{\mathrm{TC}} /\left(I_{0}-I_{\mathrm{FC}}\right)$ as a function of target retarding voltage with both nitrogen and bromocamphor targets, we can calculate $\alpha$, the component of the DEA signal due to scattered electrons, over the energy range of the data. These results are shown in Fig. 5. The smallest contributions from scattered electrons occur at energies greater than $0 \mathrm{eV}$. As the detection efficiency for negative ions of the inner target cell is unknown, the scattered-electron component is presented for several detection efficiencies. A nonunity negative ion detection efficiency is reasonable to expect and is probably due, at least partially, to the fact that our outer target cell was biased positively relative to the inner cell by a larger amount $(\sim 1.5 \mathrm{~V})$ than is typically used $(\sim 0.1 \mathrm{~V})$ [16]. Because it is not possible to have $\alpha<0$ (see Fig. 5), our results show that we detect more than $35 \%$ of the negative ions from DEA.

The energy dependence of the measured DEA asymmetries will be affected slightly by the varying component of the target cell wall current arising from elastically scattered electrons. Because the contribution from scattered electrons

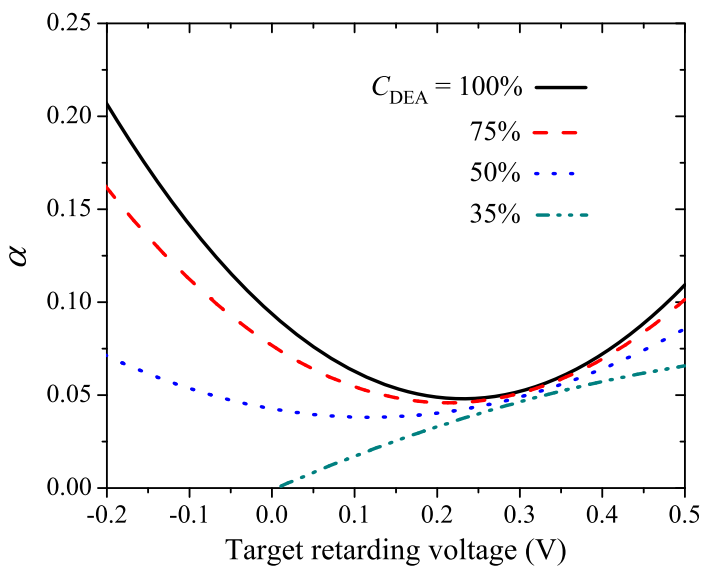

FIG. 5 (color online). Component of the inner target cell current due to scattered electrons, $\alpha$, as a function of target retarding voltage for different assumptions about $C_{\mathrm{DEA}}$. The curves represent quadratic fits to the data, which are distributed about their respective fits with a standard deviation of $\sim 0.03$.

is smallest above $0 \mathrm{eV}$, the asymmetries measured in this energy range likely represent our most accurate determination of the true chiral DEA asymmetry. The chirality of a beam of longitudinally spin-polarized electrons increases with electron velocity [28]. However, in considering the transmission of spin-polarized electrons through chiral bromocamphor [24,25], substantial asymmetries occur only at the lower electron energies, while at higher electron energies $(\sim 10 \mathrm{eV})$, the asymmetries approach zero. This suggests that for the energy range considered, resonances are a more important factor than electron chirality magnitude in determining the size of asymmetries.

To our knowledge, no calculations of chiral asymmetries involving real molecules (as opposed to simplified models) have been done. Thus, our discussion of the energy dependence of our asymmetry data must be speculative. Scattered-electron currents as well as transmitted electrons can be expected to have chiral asymmetries. Indeed, at the lowest electron energies, we might expect enhanced asymmetries for free scattered electrons because of their longer (nonresonant) interaction times with the target molecules. Thus, the sign flip and increase in the magnitude of the asymmetry for decreasing energies below $0 \mathrm{eV}$, where the contribution of scattered electrons to the target wall current is greatest, may be due in part to this scattered-electron component. If the sign of the asymmetry below $0 \mathrm{eV}$ is characteristic of these electrons, their small contribution to the asymmetries above $0 \mathrm{eV}$ would mean that these higherenergy values represent a lower limit on the true molecular breakup anion current asymmetry.

These data provide evidence for chirally sensitive, electron-induced molecular breakup in a well-defined chemical reaction. Although they tell us nothing specific about the likelihood of an actual Vester-Ulbricht mechanism operating in a prebiotic environment, they validate the 
underlying idea behind the Vester-Ulbricht hypothesis [2,3] and thus provide circumstantial evidence for this view of the origins of biological homochirality.

The idea to search for asymmetric chiral interactions in dissociative electron attachment was proposed by P. D. Burrow. We would like to thank P.D. Burrow, M. I. Fabrikant, E. T. Litaker, and K. W. Trantham for assistance with the experiment. This work was funded by the National Science Foundation, Grant No. PHY-1206067 and the NASA Nebraska Space Grant.

*jmdreiling2@gmail.com

[1] A. C. Evans, C. Meinert, C. Giri, F. Goesmann, and U. J. Meierhenrich, Chem. Soc. Rev. 41, 5447 (2012).

[2] F. Vester, T. L. V. Ulbricht, and H. Krauch, Naturwissenschaften 46, 68 (1959).

[3] T. L. V. Ulbricht and F. Vester, Tetrahedron 18, 629 (1962).

[4] D. Fitz, H. Reiner, K. Plankensteiner, and B. M. Rode, Curr. Chem. Biol. 1, 41 (2007).

[5] W. A. Bonner, Chirality 12, 114 (2000).

[6] W. A. Bonner, Origins Life Evol. Biosphere 21, 59 (1991).

[7] See, e.g., L. A. Hodge, F. B. Dunning, G. K. Walters, R. H. White, and G. J. Schroepfer, Nature (London) 280, 250 (1979).

[8] See, e.g., W. A. Bonner, N. E. Blair, and J. J. Flores, Nature (London) 281, 150 (1979).

[9] W. A. Bonner, R. M. Lemmon, and H. P. Noyes, J. Org. Chem. 43, 522 (1978).

[10] W. A. Bonner and R. M. Lemmon, Bioorganic Chemistry 7, 175 (1978).

[11] R. K. Tokay, B. Norden, J. O. Liljenzin, and S. Andersson, J. Radioanal. Nucl. Chem. 104, 337 (1986).
[12] R. A. Rosenberg, M. Abu Haija, and P. J. Ryan, Phys. Rev. Lett. 101, 178301 (2008).

[13] R. A. Rosenberg, Top. Curr. Chem. 298, 279 (2011).

[14] L. G. Christophorou, D. L. McCorkle, and A. A. Christodoulides, in Electron-Molecule Interactions and Their Applications, edited by L. G. Christophorou (Academic Press, Orlando, 1984), p. 477.

[15] T. M. Stephen, X. Shi, and P. D. Burrow, J. Phys. B 21, L169 (1988).

[16] K. Aflatooni and P. D. Burrow (private communication).

[17] B. Boudaiffa, P. Cloutier, D. Hunting, M. A. Huels, and L. Sanche, Science 287, 1658 (2000).

[18] F. Martin, P. D. Burrow, Z. Cai, P. Cloutier, D. Hunting, and L. Sanche, Phys. Rev. Lett. 93, 068101 (2004).

[19] H. Abdoul-Carime, S. Gohlke, and E. Illenberger, Phys. Rev. Lett. 92, 168103 (2004).

[20] T. M. Orlando, D. Oh, Y. Chen, and A. B. Aleksandrov, J. Chem. Phys. 128, 195102 (2008).

[21] D. T. Pierce, R. J. Celotta, G.-C. Wang, W. N. Unertl, A. Galejs, C. E. Kuyatt, and S. R. Mielczarek, Rev. Sci. Instrum. 51, 478 (1980).

[22] M. I. Fabrikant, K. W. Trantham, V. M. Andrianarijaona, and T. J. Gay, Appl. Opt. 47, 2465 (2008).

[23] T. J. Gay, J. E. Furst, K. W. Trantham, and W. M. K. P. Wijayaratna, Phys. Rev. A 53, 1623 (1996).

[24] S. Mayer, C. Nolting, and J. Kessler, J. Phys. B 29, 3497 (1996).

[25] C. Nolting, S. Mayer, and J. Kessler, J. Phys. B 30, 5491 (1997).

[26] K. Aflatooni and P. D. Burrow, J. Chem. Phys. 113, 1455 (2000).

[27] P. R. Bevington and D. K. Robinson, Data Reduction and Error Analysis for the Physical Sciences (McGraw Hill, New York, 1992), p. 58.

[28] L. D. Barron, Chem. Soc. Rev. 15,189 (1986). 